\title{
MENOPAUSA, SINTOMAS DE MENOPAUSA E DEPRESSÃO: INFLUÊNCIA DO NÍVEL EDUCACIONAL E DE OUTRAS VARIÁVEIS SOCIODEMOGRÁFICAS
}

\author{
Teresa Fagulha ${ }^{1}$ \\ Bruno Gonçalves ${ }^{2}$
}

Resumo: Este estudo descreve a sintomatologia relacionada com a menopausa nos períodos pré-menopausa, menopausa e pós-menopausa, em relação com a sintomatologia depressiva, nível educacional, estatuto conjugal e outras variáveis sociodemográficas, numa amostra de 127 mulheres com idades compreendidas entre os 35 e os 65 anos, que compareceram a uma consulta com o médico de família num Centro de Saúde dos arredores de Lisboa. As mulheres responderam a um questionário com questões relativas ao estatuto percebido de menopausa e à presença de sintomas com ela relacionados, e participaram numa entrevista clínica estruturada adaptada do módulo de avaliação das perturbações do humor da Structured Clinical Interwiew for DSM-IV Axis I Disorders (SCID-I). Descreve-se a frequência da sintomatologia relacionada com a menopausa e analisa-se a sua variação em relação com o nível educacional e outras variáveis sociodemográficas. A relação entre o estatuto percebido de menopausa e a sintomatologia depressiva é também analisada, tomando em consideração o nível educacional.

Palavras-chave: menopausa, sintomas, depressão, nível educacional, estatuto conjugal.

\begin{abstract}
Menopause, menopause symptoms and depression: The influence of educational level and other socio-demografic variables (abstract): This study is based on the data collected in an investigation regarding the Primary Health Care patients. The results relating to a sample of 127 women with ages ranging between 35 and 65 years of age are analyzed. All these women answered a Questionnaire concerning the perceived menopause status and the menopause symptomatology and participated in a structured clinical interview, in which the presence of various

\footnotetext{
${ }^{1}$ Professora Associada da Faculdade de Psicologia e de Ciências da Educação da Universidade de Lisboa. Centro de Psicologia Clínica e Experimental da Fundação para a Ciência e Tecnologia. E-mail: tfagulha@fpce.ul.pt

2 Professor Associado da Faculdade de Psicologia e de Ciências da Educação da Universidade de Lisboa. Centro de Psicologia Clínica e Experimental da Fundação para a Ciência e Tecnologia.
} 
forms of depression according to the DSM-IV criteria was analyzed. They also answered a questionnaire concerning the perceived menopause status as well as symptoms or alterations related with menopause. The frequency of the symptomatology related with menopause is studied and its variation in relation to the level of education, and other sociodemographic variables is analyzed. The relationship between the perceived menopause status and the depressive symptomatology is also studied, taking into consideration the level of education.

A meia-idade constitui uma fase de reformulações nas relações consigo mesmo e com os outros, decorrente de alterações biológicas e relacionais que ocorrem neste período e que são, nas mulheres, fortemente marcadas pelas modificações que surgem com a menopausa. A forma como a menopausa é vivida por cada mulher depende do seu funcionamento psicológico e do contexto sociocultural, mas este fenómeno biológico tem necessariamente um impacto enquanto finalização da vida reprodutiva e anúncio do envelhecimento. As queixas e transtornos que então surgem os sintomas menopáusicos - deverão ser encarados no contexto duma vivência global, que engloba as alterações endócrinas, mas também os conteúdos psicológicos associados a todas estas transformações biológicas e relacionais, bem como as características de personalidade de cada mulher.

A perda de controlo sobre o corpo, afectado por irregularidades de funcionamento, sacudido por ondas de calor súbitas, que expõem publicamente as mudanças e experiências internas, não permitindo, como Kittel e Voda (1998) assinalaram, "manter as aparências", vem precipitar a necessidade e urgência de compreensão e integração deste corpo em mudança. Tal como o psíquico tem influência na condição biológica, também os significados atribuídos pela mulher às alterações que experimenta no seu corpo precisam ser entendidos e respondidos para que ela possa integrar esta nova imagem corporal, este corpo de mulher que já não pode ter filhos e que envelhece. O confronto com esta realidade é, frequentemente, concomitante com modificações nas relações conjugais que se desgastaram, na relação com os filhos que se emancipam, e com os pais que envelhecem.

A "mudança de idade" é, muitas vezes, uma profunda mudança de vida, que suscita vivências depressivas, exigindo um trabalho de adaptação e elaboração que conduza a uma nova organização. De acordo com o estudo de MacKinley, McKinlay e Brambilla (1987), para a maioria das mulheres o aumento de depressão na menopausa está associado com essas múltiplas fontes de stress social, mais do que com factores biológicos.

De facto, durante a fase da menopausa ocorrem provavelmente mais mudanças - físicas, psicológicas, sociais e espirituais - do que em qualquer 
outro período da vida adulta da mulher. Para além das mudanças relacionais, há uma transformação no sentimento do tempo (agora um tempo em que a finitude se faz sentir) e um repensar dos aspectos negativos e positivos da transição para um estado novo e desconhecido. Com a actual esperança de vida, que favorece a ilusão de que o envelhecimento é cada vez mais algo que se pode controlar, adiar e até talvez mesmo parar (Gonyea, 1998), a menopausa inaugura um período que pode corresponder a cerca de metade do tempo da vida adulta. Quando a mulher se confronta com ela, por volta dos 50 anos, passaram três décadas desde os seus 20 anos (muitas vezes sentidos como ainda tão perto) e, muito provavelmente, tem ainda uma forte probabilidade de viver outras tantas. Trata-se, pois, de uma fase de transição que merece uma atenção particular, na medida em que proporciona reestruturações que afectam - para o melhor, ou para o pior - a forma como esse novo tempo será vivido.

O problema é tanto mais relevante quanto, em consequência do aumento de nascimentos que se verificou entre 1946 e 1964, é muito elevado o número de mulheres que, em cada ano, entram nesta fase da vida. Um relatório de 1993 do Banco Mundial (citado por Hulka \& Meirik, 1996) estima que a população de mulheres pós-menopausicas aumentará de 470 milhões em 1990 para 1,2 biliões em 2030. Tudo isto, conjuntamente com a progressiva afirmação dos "estudos femininos", conduziu a um aumento de interesse no estudo da menopausa. Os primeiros estudos, com início na década de 20, tiveram como base o chamado modelo biomédico (e.g., Greendale \& Arriola, 1999), decorrente dos avanços da endocrinologia, que foca os aspectos biológicos - o défice hormonal a repor, "tratando" os sintomas - e que procura também dar resposta a outras condições associadas aos efeitos das alterações hormonais nesta fase da vida (osteoporose, problemas cardíacos, etc.).

Do ponto de vista psicológico, este modelo tem sido fortemente contestado (e.g., Gannon \& Ekstrom, 1993; Rostosky \& Travis, 1996) por não englobar os factores psicológicos, sociais e culturais que interagem com os biológicos, e por acentuar os aspectos sintomáticos que, numa visão optimista, não estão necessariamente presentes. Surge, então, uma perspectiva desenvolvimental, defendendo que a menopausa corresponde a uma transição natural, que não ocasiona necessariamente perturbação, o que tem sido sustentado por vários estudos (e.g., Ballard, Kuh, \& Wadsworth, 2001; Gannon \& Ekstrom, 1993; MacKinley et al., 1987; Matthews et al., 1990). No entanto, e se bem que natural, é impossível negar, como atrás referimos, que esta transição funciona como um "marco" no percurso de vida, catalisando a consciência do tempo que passa e originando uma experiência de transformações corporais, psíquicas e relacionais, certamente com significados únicos para cada mulher, com o seu dinamismo 
particular e com as suas circunstâncias de vida (e.g., Amann-Gainotti \& Menci, 1993; Bush, \& Zonderman, 1994; Mendell, 1988; Ballard et al., 2001; Becker et al., 2001; Stephens, 2001), mas que frequentemente se caracteriza pela ocorrência de perturbações, sentidas com maior ou menor gravidade, e que vários estudos têm descrito.

A relevância dos aspectos socioculturais, que condicionam o modo como a mulher é olhada quando envelhece - valorizada ou desvalorizada na sua imagem e nos seus papéis sociais - e os próprios hábitos e condições de vida (e.g., suporte económico, alimentação, estatuto de saúde), tem também sido analisada. Estudos sobre os sintomas e o significado da menopausa em mulheres de diferentes culturas realçam a existência de diferenças (e.g., Im \& Meleis, 2000; Bromberger et al., 2001; Hulka \& Meirik, 1996; Lee, 1997; Lockely, 1991; Sampselle, Harris, Harlow, \& Sowers, 2002). Nas culturas ocidentais, orientadas para o culto da juventude, a menopausa é frequentemente associada a medo de envelhecimento, perda de estatuto e problemas com a sexualidade, o que não tem paralelo em sociedades com valores culturais diferentes. Assim, Christiane Northrup (2000) refere que, em algumas culturas nativas, as mulheres só podem ser "xamã" depois de ter ultrapassado a menopausa, pois só então retêm o "sangue da sabedoria", que lhes dá a possibilidade de deter grande poder, tomar decisões e responsabilidade em relação às crianças e às mulheres mais novas. Ainda segundo a mesma autora, nas culturas celtas, a rapariga jovem era vista como a flor; a mãe, como o fruto; e a mulher mais velha como a semente, ou seja, a parte que contém dentro de si o conhecimento e o potencial de todas as outras partes. É ainda ela que nos diz que na China, onde a idade é venerada, as mulheres não apresentam queixas relacionadas com a menopausa.

Num estudo sobre o significado cultural das categorizações e interpretações acerca das mudanças biológicas relacionadas com a menopausa, focando a construção cultural da menopausa no Japão, Lockely (1991) refere a dificuldade de tradução do termo afrontamento (necessitou utilizar três palavras em conjunto para expressar a ideia), por não existir na língua japonesa uma palavra com este significado, se bem que existam termos referentes a subtis distinções de estados corporais. Poderíamos encarar este facto como sinal de um tabu relativo à sexualidade feminina, tabu que, de facto, se faz sentir de um modo particular em relação a este acontecimento particular da vida da mulher, mas Lockely (1991) constata que quer as mulheres, quer os médicos japoneses têm uma perspectiva de transição natural, relacionada com o envelhecimento, em que os sintomas especificamente relacionados com as alterações endócrinas, como os afrontamentos, têm uma ocorrência irrelevante, encarando-os como o resultado de alterações do sistema nervoso autónomo. $\mathrm{O}$ facto de as japonesas, quando 
comparadas com as mulheres ocidentais, apresentarem um número muito menor de queixas e problemas de saúde relacionados com a menopausa, (são muito menores os problemas de osteoporose, cardíacos e de cancro da mama) pode ser explicado pelos hábitos alimentares - uma dieta muito rica em peixe, com altos níveis de cálcio e de soja (uma fonte natural de estrogénio) - e pelo exercício regular: bicicleta e caminhada (Northrup, 2000). Greendale, Lee e Arriola (1999) referem que a percentagem de afrontamentos pode variar entre $80 \%$, nos países ocidentais e $10 \%$ em países da Ásia oriental. Numa análise de vários estudos sobre a frequência de sintomas associados à menopausa, também Hulka e Meirik (1996) encontram enormes diferenças na ocorrência de afrontamentos: $80 \%$ nas holandesas, $50 \%$ nas norte-americanas, $12 \%$ nas japonesas e $0 \%$ nas mulheres da cultura Maya da América Central.

Estudos com mulheres negras têm revelado que também elas experimentam menos perturbação relacionada com a menopausa, quando comparadas com as brancas. Uma explicação possível pode residir no facto de não valorizarem tanto a sexualidade e feminilidade através do aspecto físico, mas essencialmente, pela força espiritual. As mulheres afro-americanas que participaram num estudo qualitativo (Sampselle et al., 2002) encaram a menopausa como uma transição esperada e natural, não associada a especial perturbação (se bem que reconhecendo o estereótipo negativo) e algumas consideraram mesmo que estavam a viver um "pico" na sua vida sexual. Neste sentido aponta o estudo de Cain e colaboradores (2003), que compara o funcionamento sexual (incluindo a frequência, a motivação expressar amor, procurar prazer, por proposta do parceiro, para aliviar a tensão - e as práticas sexuais), numa larga amostra de mulheres americanas de cinco grupos étnicos na pré e perimenopausa. Estes autores encontram uma disparidade na frequência da relação sexual - mais alta nas afro-americanas do que nas caucasianas, enquanto que as japonesas se distinguem destas últimas por uma menor frequência.

Ao entrevistar várias mulheres americanas, Gail Sheehy (1994) verificou que as que desempenham papéis nos quais a actividade intelectual, a criatividade ou a força espiritual são valorizadas (artistas, políticas, escritoras, terapeutas) lidam melhor com a transição da menopausa, enquanto que as mulheres cujo valor sempre dependeu mais da sua aparência física ou do seu papel na família (actrizes, mulheres só mães e donas de casa) se sentem mais diminuídas no seu estatuto e parecem mais predispostas à depressão nesta fase da vida. No mesmo sentido, conclui Nadelson (citado no Final Report da American Psychological Association's National Task Force on Women and Depression -, McGrath, Keita, Strickland \& Russo,1995), que, na revisão da literatura sobre este tema, encontra maior experiência de perturbação na menopausa nas mulheres que investiram fundamentalmente 
no papel de mães como forma de reconhecimento e fonte de auto-estima. Esse mesmo relatório faz ainda referência às diferenças de sintomatologia menopausica, de acordo com o estatuto social: as mulheres de classe média e média-alta diferenciam-se das mulheres de classes desfavorecidas por considerarem que a menopausa representa uma libertação que lhes abre novas oportunidades, finalizadas as tarefas de cuidado dos filhos, o que permite uma experiência mais positiva. O estudo de Sampselle e colaboradores (2002) atrás referido, realizado com uma amostra de mulheres afro-americanas e de mulheres brancas, empregadas e de classe média, encontra confirmação deste ponto de vista. Utilizando uma metodologia de discussão em grupo, os autores referem que não sentem que o crescimento e emancipação dos filhos represente uma perda, mas antes uma oportunidade de investimento pessoal e auto-reflexão: as mulheres têm, então, mais tempo para si próprias, para poderem pensar em si, em vez de se porem sempre em último lugar, o que lhes permite maior autenticidade e mais consciência do seu valor. Não deixa, no entanto, de ser interessante notar que estas umlheres consideraram que o facto de se sentirem mais confiantes e com mais auto-estima, menos dependentes das opiniões e expectativas dos outros, era encarado como um sinal negativo, de "menopausa", pelos seus companheiros, que não estavam habituados a que as mulheres pensassem em si próprias.

Podemos, então, perguntar-nos: e entre nós, como é que as mulheres reagem a esta transição? Verificamos, na clínica, que é cada vez maior o número de mulheres de meia-idade a procurar ajuda, queixando-se de perturbações cujo significado precisam entender para que possam empreender reformulações que conduzam a novos equilíbrios e a novos investimentos. Desconhecemos a existência de estudos que descrevam os sintomas mais comuns.

Vários estudos noutros países têm analisado os sintomas que ocorrem nesta fase: os sintomas específicos, que decorrem directamente da diminuição dos níveis hormonais de estrogénio e progesterona, como afrontamentos e suores nocturnos (os chamados sintomas vasomotores) e a atrofia do aparelho urogenital, que pode originar secura vaginal, incontinência urinária, infecções do tracto urinário (Greendale \& Arriola, 1999). E os sintomas não específicos, porque podem ocorrer em qualquer outra fase de transição, em consequência do stress - alterações de humor (irritabilidade, ansiedade e sintomas depressivos), problemas de sono e de memória, fadiga, diminuição da libido, dores de cabeça, etc. (o próprio número e variabilidade dos sinais denuncia a sua não especificidade). De notar que num estudo comparativo dos sintomas não específicos (Van Hall et al., 1994, citado por Hulka \& Meirik, 1996) não se encontraram diferenças entre as queixas apresentadas pelos homens e pelas mulheres neste período da vida. 
A ocorrência frequente dos sintomas não específicos é encarada por alguns autores como estando fortemente associada à experiência dos sintomas vasomotores. Por exemplo, pode entender-se que quando a mulher acorda com os suores nocturnos, o sono REM é interrompido. A mulher, acordada por alterações de temperatura e privada do sono REM necessário à manutenção do termóstato corporal, é presa dum ciclo vicioso, que fragiliza o seu funcionamento psicológico e a torna mais vulnerável às alterações de humor, mais facilmente irritável. Bromberger e colaboradores (2001) encontram, de facto, uma forte relação entre os problemas de sono e a perturbação psicológica, bem como entre os sintomas vasomotores e a perturbação psicológica. No entanto, quando incluem os sintomas vasomotores e as perturbações de sono em análises multivariadas, não é eliminada a relação entre o estatuto de menopausa e a perturbação psicológica, o que sugere que os sintomas vasomotores e os problemas de sono não explicam completamente esta relação, sugerindo que o estatuto de menopausa poder ser um contribuinte independente para a referida perturbação.

Os estudos iniciais sobre sintomatologia menopausica utilizaram amostras clínicas, como é o caso do nosso estudo. Suscitaram críticas, na medida em que a extrapolação dos resultados a partir de amostras constituídas por mulheres que procuram tratamento "ajudou a criar um estereótipo da mulher menopausica como tendo uma variedade de sintomatologia difusa que precisa de cuidado médicos" (Final report da American psychological association's national task force on women and depression, McGrath et al., 1995, p. 13). Rostosky e Travis (1996) questionam a própria terminologia utilizada, na medida em que a palavra sintoma tem uma conotação médica, associando a menopausa a doença, visão a que se opõem, defendendo, como atrás referimos, uma óptica de transição natural.

Para entender o modo como as mulheres saudáveis reagem à menopausa, desenvolveram-se, então, nos Estados Unidos e em Inglaterra, investigações que utilizaram amostras comunitárias, abrangendo largas faixas da população, algumas das quais incluíram minorias étnicas (e.g., Bromberger et al., 2001), bem como estudos longitudinais que permitiram acompanhar as mulheres durante toda a fase de transição - da pré-menopausa ao período pós-menopausa. Inseridos em projectos alargados sobre a saúde da mulher, estes estudos envolveram grandes equipas. Como exemplos, temos o Study of Women's Health Across the Nation, abreviadamente designado por SWAN, em que se insere o estudo acima citado de Bromberger e colaboradores e o estudo de Sampselle e colaboradores (2002), que utiliza uma metodologia qualitativa de grupo; o National health examination Follow-up Study, estudo longitudinal em que se inseriu o estudo de Bush e Zonderman (1994), que utiliza uma amostra de 2.268 mulheres; o Massachussetts women's health study, iniciado em 1981, e que 
incluiu uma amostra de mais de 2.500 mulheres; o estudo longitudinal de Mathews e colaboradores (1990), com uma amostra de 541 mulheres com um bom estatuto de saúde; o medical research council national survey of health and development, em que se insere o estudo de Ballard e colaboradores (2001); o midlife women's health survey, estudo longitudinal com mulheres predominantemente de raça branca e com bom nível sócioeconómico, em que se inseriu o estudo de Mansfield e Voda (1997).

Procurando integrar os aspectos bio-psico-sociais, a maioria destes estudos analisou a relação entre os sintomas de menopausa e variáveis sociodemográficas ou psicológicas, encontrando uma complexidade de interacções entre essas variáveis e a ocorrência de perturbação. Algumas dessas variáveis - como as expectativas em relação ao envelhecimento, as características de funcionamento psicológico, os factores de stress psicológico, o estatuto de saúde - podem atenuar as diferenças encontradas em função dos contextos socioculturais. Como exemplo, podemos referir que Bromberger e colaboradores (2001) encontram menor frequência de sintomas de perturbação nas mulheres chinesas e japonesas, em relação às mulheres de raça branca, mas estas diferenças esbatem-se quando se consideram variáveis como o suporte financeiro e social e o estatuto de saúde.

A escassez de recursos não nos permitiu ultrapassar a utilização de uma amostra de dimensões reduzidas, uma amostra clínica que se insere num projecto mais vasto sobre a ocorrência de depressão na mulher de meia-idade.

O presente estudo, de carácter exploratório, tem como objectivos: 1) Avaliar a frequência dos vários sintomas ou alterações relacionados com a menopausa, numa amostra de mulheres portuguesas, e as eventuais variações desta sintomatologia em função do estatuto percebido de menopausa; 2) Analisar a variação desta sintomatologia em função de variáveis sociodemográficas, como a idade, a escolaridade, estar casada, empregada, ter filhos, ter filhos a cargo, ter filhos em casa; 3) Analisar a relação entre o estatuto de menopausa, as variáveis sociodemográficas e a sintomatologia depressiva.

\section{Método}

\section{Participantes e recrutamento}

O presente estudo insere-se no âmbito duma investigação mais global sobre a prevalência de depressão na mulher de meia-idade. Durante um período de três meses, todas as mulheres com idades compreendidas entre 
os 35 e os 65 anos, que compareceram no Centro de Saúde para consultar o médico de família, foram convidadas a participar num projecto de investigação, a decorrer com a colaboração do referido Centro e publicitado em cartazes. As mulheres que aceitaram colaborar responderam aos questionários no momento em que se solicitava a participação, e acordaram a data para a realização da entrevista clínica.

\section{Procedimento}

Aplicação de um Questionário de Dados Sociodemográficos elaborado para o estudo (estatuto conjugal: solteira, casada / união de facto, separada/divorciada, viúva; número de anos de escolaridade: menos de 4 anos, 4 anos, 6 anos, 9 anos, 12 ou mais anos; número de filhos; número de filhos ainda a cargo, número de filhos ainda em casa; estatuto laboral: empregada, desempregada, dona de casa, reformada, com baixa médica. $\mathrm{O}$ questionário abrangeu ainda outras variáveis sociodemográficas (que constam do estudo de Gonçalves \& Fagulha, neste número).

O estatuto percebido de menopausa foi avaliado através de uma pergunta incluindo três alternativas: "ainda não tenho problemas de menopausa", "tenho actualmente problemas de menopausa", "já ultrapassei os problemas de menopausa". Aplicação de um Questionário de Sintomas ou Alterações que as mulheres relacionassem com a menopausa, (afrontamentos, suores nocturnos, alteração da aparência física, irritação, problemas de sono, alteração do desejo sexual - aumento/diminuição). A avaliação clínica foi ainda utilizada para a construção de um índice de intensidade de sintomatologia depressiva (ver artigo de Gonçalves \& Fagulha, nesta revista).

Entrevista clínica semiestruturada, adaptada do módulo de avaliação das perturbações do humor da versão clínica da Structured Clinical Interview for DSM-IV Axis I Disorders (SCID-I; First et al., 1997), para avaliar a presença de perturbação depressiva, de acordo com os critérios do DSM-IV.

\section{Amostra}

129 mulheres responderam às perguntas sobre menopausa e 127 aceitaram participar na entrevista. A média de idades das mulheres é de 51,4 $(\mathrm{SD}=8,9)$. A amostra divide-se em 3 grupos, de acordo com o estatuto percebido de menopausa: 47 mulheres $(36 \%$, ) com 42,4 $\pm 5,3(\mathrm{M} \pm$ DP) anos de idade foram incluídas na pré-menopausa; 28 mulheres $(22 \%)$ com 51,2 $\pm 5,9$ anos de idade $(\mathrm{M} \pm \mathrm{DP})$ foram incluídas na menopausa; 54 mulheres $(42 \%)$ com $58,9 \pm 4,6$ anos de idade $(\mathrm{M} \pm \mathrm{DP})$ foram incluídas na pós-menopausa. Em relação ao estatuto conjugal: $14 \%$ são solteiras; $65 \%$ 
casadas; $16 \%$ são divorciadas ou separadas; $6 \%$ são viúvas. A distribuição do nível educacional, de acordo com o número de anos de escolaridade; é a seguinte: menos de 4 anos: 12\%; 4 anos: $31 \%$; 6 anos: 22\%; 9 anos: 17\%; 12 ou mais anos: $10 \%$. No que se refere à situação laboral: $57 \%$ estão empregadas; $13 \%$ desempregadas; $12 \%$ reformadas; $13 \%$ são donas de casa; e 5\% estão com baixa médica. No que respeita aos filhos, $16 \%$ não têm filhos; $56 \%$ têm filhos ainda em casa - 44\% ainda dependentes e 12\% já independentes; $28 \%$ têm os filhos já independentes e vivendo fora de casa.

\section{Resultados}

O número médio de sintomas ou alterações referidos pelas mulheres na menopausa é de 2,21 contra 1,24 nas mulheres na pós-menopausa. Os sintomas mais frequentes são os vasomotores e a alteração da aparência física: afrontamentos $(50 \%)$, suores nocturnos (43\%), alterações da aparência física (43\%), tendo os sintomas não específicos percentagens de ocorrência mais baixas: irritação (35\%), diminuição de libido (29\%), perturbações de sono (14\%) e aumento de libido (7\%). À excepção do aumento de libido, todos os sintomas se encontram também, embora com menor frequência, na pós-menopausa. Neste período, os sintomas mais frequentes são ainda os vasomotores: afrontamentos (31\%) e suores nocturnos (30\%), alteração da aparência física (26\%), irritação (17\%), diminuição de libido $(13 \%)$ e perturbações de sono $(7 \%)$.

O Quadro 1 mostra como estes sintomas e alterações se correlacionam entre si. Não incluímos neste quadro o aumento de libido, dado que é relativamente raro.

Quadro 1: Correlações entre os sintomas relacionados com a menopausa

\begin{tabular}{lccccc}
\hline & Afrontamentos & Suores & Irritação & Sono & Aparência \\
\hline Afrontamentos diurnos & - & & & & \\
Suores nocturnos & $0,55^{*}$ & & & & \\
Irritação & $0,29^{*}$ & 0,15 & & & \\
Alterações do sono & $-0,00$ & 0,11 & 0,21 & & \\
Alterações da aparência & 0,06 & 0,12 & $0,25^{*}$ & 0,13 & \\
Diminuição de libido & 0,15 & $-0,01$ & 0,15 & $0,27^{*}$ & 0,15 \\
\hline
\end{tabular}

$* \mathrm{p}<0,05$ 
A fim de avaliar o eventual efeito das variáveis sociodemográficas na sintomatologia relacionada com a menopausa, foram utilizadas estatísticas univariadas (U de Mann-Whitney) para comparar o número de sintomas relacionados com a menopausa referidos pelas mulheres com diferentes características sociodemográficas. Uma primeira exploração dos dados não permite encontrar diferenças significativas, mas sugere que poderá ser útil ter em conta a interacção das diferentes características com a escolaridade. Assim, no nível sociocultural mais baixo, as mulheres casadas referem, em média, menos sintomas de menopausa do que as outras (1,3 contra 2,3). Esta diferença, que é apenas tendencialmente significativa $(\mathrm{p}=0,079)$, não se verifica nas mulheres de nível cultural mais elevado, onde os valores correspondentes são ambos de 1,6.

Quadro 2: Influência de variáveis sociodemográficas na ocorrência de afrontamentos diurnos (análise de regressão logística descendente stepwise)

\begin{tabular}{lccc}
\hline Variáveis & Sig.* & RP & $\begin{array}{c}\text { Intervalo de confiança a 95\% } \\
\text { para OR }\end{array}$ \\
\hline Menopausa & 0,023 & 4,53 & {$[1,23 ; 16,70]$} \\
Casada & 0,047 & 0,30 & {$[0,09 ; 0,99]$} \\
Escolaridade x Menopausa & 0,074 & 0,18 & {$[0,03 ; 1,18]$} \\
Escolaridade x Casada & 0,095 & 3,27 & {$[0,81 ; 13,12]$} \\
\hline
\end{tabular}

* Significância do coeficiente de Wald

Procedemos a uma análise de regressão logística, utilizando um método descendente stepwise, introduzindo como variável dependente a ocorrência de afrontamentos diurnos (sintoma mais frequente) e, como variáveis independentes, o estatuto de menopausa, a idade, a escolaridade, estar casada, empregada, ter filhos, ter filhos a cargo, ter filhos em casa. Considerámos ainda a interacção de cada uma destas variáveis com a escolaridade. Foram incluídas as mulheres na menopausa e na pós-menopausa $(\mathrm{n}=82)$. O modelo final é apresentado no Quadro 2. Para a interpretação dos resultados convém ter presente que, na presença de um termo relativo à interacção entre duas variáveis, o efeito isolado de cada uma delas é avaliado para o nível zero da outra. O valor de odds ratio (OR) inferior a 1 indica que o factor em questão diminui a probabilidade de ocorrência de afrontamentos. Mais uma vez se verifica que, nas mulheres de nível sociocultural baixo, o facto de estar casada tende a diminuir a ocorrência de afrontamentos, sintoma que é, simultaneamente, mais referido por estas mulheres. Além disso, como seria de esperar, o facto de 
estar na menopausa aumenta a probabilidade de ocorrência de afrontamentos (relativamente à pós-menopausa). Mas, curiosamente, isso parece só se verificar nas mulheres de baixo nível sociocultural. Os efeitos das outras variáveis são apenas tendencialmente significativos: sugerem que as mulheres de nível sociocultural alto tendem a referir menos afrontamentos na menopausa (relativamente à pós-menopausa) e que as mulheres casadas de nível sociocultural alto tendem a referir mais afrontamentos.

Interessava-nos especialmente estudar a relação entre menopausa e depressão. O Quadro 3 apresenta a média de sintomas depressivos, avaliados na entrevista clínica, em função do estatuto de menopausa. O teste de análise de variância não paramétrica de Kruskal-Wallis mostra que o efeito do estatuto de menopausa é tendencialmente significativo $(p=0,056)$. Tendo em atenção que o número de sintomas depressivos é influenciado pelo estatuto sociocultural (ver artigo de Gonçalves \& Fagulha, nesta revista), calculámos os mesmos valores separadamente para o subgrupo de baixo nível de instrução e para o subgrupo de nível de instrução mais elevado. Estes valores sugerem que o aumento da sintomatologia depressiva na menopausa é, sobretudo, evidente no grupo de nivel de instrução baixo. O teste de Kruskal-Wallis é, neste subgrupo, muito significativo $(\mathrm{p}=0,0004)$ e não é significativo no subgrupo de nível de instrução mais elevado $(\mathrm{p}=0,273)$.

Quadro 3: Estatuto de menopausa e média de sintomas depressivos

\begin{tabular}{lccc}
\hline & Total & $\begin{array}{c}\text { Menos de 9 anos de } \\
\text { escolaridade }\end{array}$ & $\begin{array}{c}\text { 9 ou mais anos de } \\
\text { escolaridade }\end{array}$ \\
\hline Pré-menopausa & 4,9 & 6,6 & 3,8 \\
Menopausa & 6,3 & 7,7 & 4,8 \\
Pós-menopausa & 4,2 & 3,2 & 5,6 \\
\hline
\end{tabular}

No subgrupo de nível de instrução mais elevado parece haver uma tendência ao aumento da sintomatologia depressiva tanto da pré-menopausa para a menopausa, como da menopausa para a pós-menopausa - o que poderá relacionar-se com a idade e não com o estatuto de menopausa. A comparação dos vários subgrupos com o teste U de Mann-Whitney indica diferenças estatisticamente significativas na comparação entre as mulheres na menopausa e as mulheres na pós-menopausa, no grupo total $(\mathrm{p}=0,016)$ e no subgrupo de baixa escolaridade $(0,0001)$. A comparação, no grupo total, entre as mulheres na pré-menopausa e na menopausa é tendencialmente significativa $(p=0,070)$. As outras diferenças não são estatisticamente significativas. 
A interacção entre o nível de instrução e o estatuto de menopausa aparece também claramente quando não se considera como variável dependente o número de sintomas depressivos, mas antes o diagnóstico de alguma forma de perturbação depressiva através da entrevista clínica (Figura 1).

Figura 1: Perturbações depressivas (em \%) em função da menopausa e da escolaridade

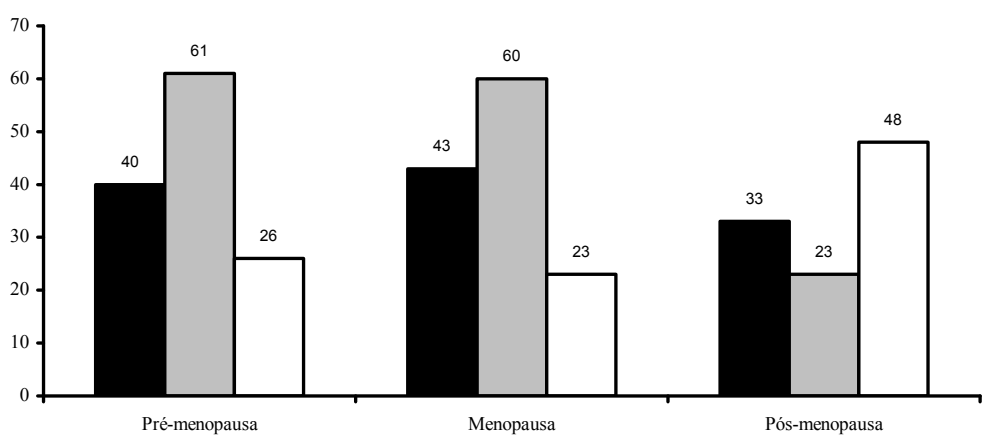

Total $\square$ Menos de 9 anos de escolaridade $\square 9$ ou mais anos de escolaridade

A interpretação dos resultados desagregados em função do nível de escolaridade levanta problemas já que, tendo em atenção a elevada correlação $(r=0,82)$ entre a idade e o estatuto de menopausa, as variações observadas poderiam igualmente ser atribuídas à idade. Uma primeira exploração dos dados recorrendo a técnicas univariadas (U de Mann-Whitney) sugere que o efeito da variável idade é apenas significativo no grupo de mulheres na pós-menopausa. Com efeito, neste subgrupo, as mulheres com mais de 59 anos (mediana do grupo) apresentam significativamente mais sintomas depressivos. Embora esta diferença só seja estatisticamente significativa quando se considera o total do subgrupo, os resultados são semelhantes nas mulheres de nível de instrução baixo e nas mulheres de nível de instrução alto. Utilizando o mesmo procedimento para as mulheres na pré-menopausa ou na menopausa, não se encontram diferenças significativas.

Para tentar avaliar mais rigorosamente o eventual efeito da variável idade tendo em conta a escolaridade e o estatuto de menopausa, foi utilizada uma regressão logística recorrendo a um método descendente stepwise, tomando a ocorrência de alguma forma de perturbação depressiva como variável dependente e introduzindo, como variáveis independentes, a idade e as variáveis categoriais relativas à escolaridade e ao estatuto de menopausa e considerando ainda a interacção entre o estatuto de menopau- 
sa e a idade e o estatuto de menopausa e a escolaridade. A classe de referência relativamente ao estatuto de menopausa é a das mulheres na pré-menopausa. O resultado final, apresentado no Quadro 4, permite verificar que as mulheres com mais escolaridade correm menos risco de depressão. Mas existe, simultaneamente, uma interacção significativa entre a escolaridade e a pós-menopausa - o que corresponde ao facto de as mulheres de nível de instrução alto correrem aparentemente um risco mais baixo de depressão na pré-menopausa e um risco muito mais elevado de depressão na pós-menopausa. O efeito da variável idade aparece apenas quando se considera a interacção entre a idade e o estatuto de menopausa, em particular na pós-menopausa, isto é, na pós-menopausa, o aumento de idade tende a elevar o risco de depressão. Finalmente, tendo em conta o efeito de todas estas variáveis, o estatuto de pós-menopausa tende a diminuir o risco de depressão.

Quadro 4: Sintomatologia depressiva, em função da escolaridade, do estatuto de menopausa e da idade (análise de regressão logística, stepwise, descendente)

\begin{tabular}{lccc}
\hline Variáveis & Sig.* & OR & $\begin{array}{c}\text { Intervalo de confiança } \\
\text { a 95\% para OR }\end{array}$ \\
\hline Escolaridade & & & {$[0,062 ; 0,801]$} \\
Estatuto de menopausa & 0,021 & 0,233 & \\
- Menopausa & 0,028 & & 0,540 \\
- Pós-menopausa & 0,084 & {$[0,000 ; 233,989]$} \\
Idade x Estatuto de Menopausa & 0,051 & & {$[0,000 ; 0,024]$} \\
- Idade x Menopausa & 0,543 & 1,048 & {$[0,902 ; 1,218]$} \\
- Idade x Pós-menopausa & 0,018 & 1,246 & {$[1,038 ; 1,495]$} \\
Escolaridade x Estatuto de Menopausa & 0,003 & & \\
- Escolaridade x Menopausa & 0,985 & 0,981 & {$[0,119 ; 8,072]$} \\
- Escolaridade x Pós-menopausa & 0,002 & 17,934 & {$[2,908 ; 110,611]$} \\
\hline
\end{tabular}

* Significância do coeficiente de Wald

\section{Discussão}

A frequência de sintomas detectados encontra paralelo em estudos anteriores (e.g., Bosworth et al., 2001; Bromberger et al., 2001; Hunter \& O'Dea, 2001), o primeiro dos quais utilizou um tipo de pergunta semelhante para classificar o estatuto percebido de menopausa. Os sintomas 
vasomotores (afrontamentos e suores nocturnos), especificamente relacionados com a diminuição dos níveis hormonais, são os mais frequentemente referidos. No presente estudo não foi avaliada a sua gravidade, ou seja, o nível em que afectam a mulher, aspecto a ter em conta em estudos posteriores.

A referência à alteração da aparência física parece reflectir a consciência das transformações corporais que acompanham o processo de envelhecimento e que a menopausa assinala (Amann-Gainotti \& Menci, 1993; Kittel et al., 1998; Stephens, 2001). O estudo transcultural de Sampselle e colaboradores (2002), com mulheres afro-americanas e mulheres brancas, e que utilizou uma metodologia de discussão em grupo, elucidou a preocupação com os sinais físicos de envelhecimento nas mulheres de raça branca, que estabeleceram uma associação imediata entre menopausa e parecer mais velha, reconhecendo o conflito que é imposto pelo ideal de juventude da sociedade ocidental, que contrasta com o aumento de peso, o cabelo que embranquece, as rugas que se acentuam.

Dos sintomas não específicos, ressaltam a irritação e as perturbações de sono. É interessante notar que a irritação se correlaciona com os mais frequentes sinais de perturbação: os afrontamentos e a alteração da aparência física (Quadro 1), não aparecendo correlacionada com as alterações de sono, como outros estudos sugerem. Estas correlacionam-se com a diminuição de libido. Este sintoma, muito frequente, é associado por alguns autores à diminuição dos níveis hormonais, mas estudos recentes, incluindo amostras com diferentes grupos étnicos, questionam o seu significado, cuja compreensão deve ultrapassar a explicação biológica para integrar uma visão que abranja a própria história relacional da mulher (Ballinger, 1989). Cain e colaboradores (2003), numa amostra de mais de três mil mulheres americanas, na pré e perimenopausa, e que incluiu 5 grupos étnicos, chegaram à conclusão que a maioria das mulheres, nesta fase da vida, considera a relação sexual importante, e o principal motivo para não terem uma actividade sexual é a falta de parceiro, o que foi igualmente apontado por Christine Laznik Idrissi el Ganouni, na sua tese de doutoramento (2002). Esta autora foca o papel fundamental do olhar do parceiro para sustentar a imagem feminina da mulher que envelhece, permitindo-lhe continuar a sentir-se desejada e desejante. Os aspectos ligados à qualidade da relação conjugal e à satisfação sexual não foram explorados no nosso trabalho, mas são o foco do artigo de Katherine Fielder e Sharon Kurpius, nesta mesma revista.

Dum ponto de vista psicanalítico, a renúncia à sexualidade nesta fase da vida parece um fenómeno bastante mais complexo, na medida em que pode também ser "observada em mulheres cujo marido permanece desejante" (Laznik, 2003, p. 14). A explicação psicanalítica, já apontada por 
Freud (1978/1912), é retomada por Helen Deutsch (1967), que fala de um aumento de libido nesta fase, em paralelo com o que ocorre na puberdade. É interessante verificar que, embora numa percentagem pequena, esse aumento de libido surge na nossa amostra. A dificuldade em lidar com o acréscimo libinal liga-se, como na puberdade, a fantasias incestuosas inconscientes - então dirigidas ao pai e agora ao filho adulto, ou a figuras filiais -, fantasias assustadoras que conduzem ao abdicar da sexualidade. Esta explicação encontra suporte no mito de Jocasta (Laznik, 2003).

A análise da influência das variáveis sociodemográficas sobre a sintomatologia relacionada com a menopausa só permitiu estabelecer uma diferença estatisticamente significativa: o facto de estar casada parece estar ligado a uma diminuição da sintomatologia menopausica, no caso das mulheres de nível educacional mais baixo, se bem que estas mulheres refiram mais afrontamentos na menopausa, quando comparadas com as mulheres de nível educacional alto. Em relação a estas, a sua frequência aumenta, pelo contrário, nas mulheres casadas. Não dispomos de informação (nomeadamente, a referente à satisfação conjugal) que nos permita uma interpretação clara destas diferenças, que apontam influência diferente do estatuto conjugal nas mulheres de diferentes níveis educacionais. Fica-nos a hipótese de que esta diferença poderá reflectir um acréscimo de dificuldades nas mulheres solteiras, viúvas e divorciadas com piores condições socioeconómicas (encarando o nível educacional como um bom indicador do nível socioeconómico). Em geral, o número relativamente reduzido de mulheres em determinadas categorias condiciona fortemente a análise estatística.

Os resultados são bastante mais sugestivos no que diz respeito à relação entre menopausa e depressão. A média de sintomas depressivos, avaliados na entrevista clínica (Quadro 3) aumenta na amostra total na fase da pré-menopausa para a menopausa, diminuindo na pós-menopausa, resultado concordante com a grande maioria dos estudos. Como referimos na introdução, nalguns estudos com amostras comunitárias este aumento não se verifica (e.g., Bush \& Zonderman, 1994).

É importante, neste caso, considerar a interacção com o nível de escolaridade. Lembre-se que o relatório final da American psychological association's national task force on women and depression (McGrath et al., 1995) foca a associação entre as condições desfavoráveis de vida e a depressão, a qual é concordante com estudos posteriores. Bosworth e colaboradores (2001) encontraram mais sintomas depressivos nas mulheres com menor nível de escolaridade e piores condições económicas. Vários estudos confirmam a relevância da depressão anterior como indicador da possibilidade de ocorrência subsequente de depressão na menopausa, em conjunto com condições de vida menos favoráveis (e.g., Amore et al. 2003; 
Hunter, 1990). Este último autor verificou, num estudo longitudinal, que a depressão anterior, juntamente com factores cognitivos e sociais, contribui com mais de $50 \%$ da variância nos sintomas depressivos referidos pelas mulheres na menopausa. De facto, na nossa amostra, verificamos um maior número de sintomas depressivos nas mulheres de nível de escolaridade baixo, quer na pré-menopausa, quer na menopausa (Quadro 3), (situação que se inverte na fase pós-menopausa, e o aumento da sintomatologia na passagem para a menopausa atinge uma diferença com valor estatístico, o que não acontece no grupo de escolaridade mais alta).

A situação inverte-se na passagem da menopausa para a pós-menopausa. Na nossa amostra, verifica-se uma nítida diminuição da sintomatologia depressiva nas mulheres de nível sociocultural baixo, variação que parece relacionável com o período crítico da menopausa. Por outro lado, a diminuição da sintomatologia depressiva na pós-menopausa não parece decorrer apenas do facto de certos sintomas de menopausa poderem ser erroneamente considerados como sintomas depressivos, já que se verifica igualmente uma diminuição nítida da percentagem de casos em que foi diagnosticada alguma forma de perturbação depressiva (Figura 1).

No caso das mulheres de nível de escolaridade mais elevado, a sintomatologia depressiva atinge, pelo contrário, um máximo na pós-menopausa (Quadro 3). Neste grupo, ocorre ainda um aumento da percentagem de casos em que foi diagnosticada alguma forma de perturbação depressiva (Figura 1), o que sugere uma menor importância do efeito da menopausa na sintomatologia e um eventual efeito da variável idade. Esta poderá traduzir a influência das representações sociais ligadas à perda de juventude e ao envelhecimento, provavelmente com menor peso para as mulheres que se confrontam com situações sociais mais desfavorecidas.

O modelo obtido deve ser considerado como essencialmente descritivo: só outros estudos com amostras mais importantes permitirão verificar até que ponto é generalizável. $\mathrm{O}$ aspecto talvez mais interessante é a importância da interacção entre o nível socioeconómico (indicado pela escolaridade) e a sintomatologia, nomeadamente a sua diminuição na pós-menopausa. Uma melhor compreensão desta interacção implica o estudo de outras variáveis que, como referimos, podem afectar o modo como a menopausa é vivida por mulheres de diferentes níveis socioeconómicos e culturais.

Uma outra limitação, já referida, diz respeito à utilização duma amostra clínica, que não pode ser considerada representativa da população geral. Uma última diz respeito ao facto de as perguntas relativas ao estatuto de menopausa não permitirem identificar o período da perimenopausa - o período imediatamente anterior à menopausa, em que se manifestam irregularidades e alterações dos ciclos e fluxo menstruais - e que é, segun- 
do a maioria dos estudos sobre a sintomatologia, aquele em que ela é mais intensa (McKinlay, 1996), de tal modo que Sheehy (1994) fala do "pânico da perimenopausa".

Apesar das referidas limitações, consideramos que este estudo exploratório poderá ajudar a conhecer a forma como as mulheres portuguesas experimentam a transição da menopausa, contribuindo para facilitar a sua compreensão, numa perspectiva clínica e preventiva (Huffman \& Myers, 1999) que se revela tão necessária quanto ainda pouco disponível entre nós.

\section{Referências}

Amann-Gainotti, M., \& Menci, A. M. (1993). Vécus corporels du sujet féminin de l'adolescence à la ménopause. Psychologie Europe, 3 (2), 43-51.

Amore, M., Di Donato, P., Papalini, A., Berta, A., Palareti, A., Ferrari, G., Chirico, C., \& De Aloysio, D. (2003). Psychological status at the menopausal transition: An Italian epidemiological study.

Ballard, K. D., Kuh, D. J., \& Wadsworth, M. E. J. (2001). The role of the menopause in women's experiences of the 'change of life'. Sociology of Health \& Illness, 23 (4), 397-424.

Ballinger, C. B. (1989). Aspectos psiquiátricos da menopausa. Psiquiatria na Prática Médica, 2 (3), 19-24.

Becker, D., Lomranz J., Pines, A., Shmotkin, D., Nitza, E., BennAmitay, G., \& Mester, R. (2001). Psychological distress around menopause. Psychosomatics, 42, 252-257.

Bosworth, H. B., Bastian, L. A., Kuchibhatla, M. N., Steffens, D. C., McBride, C. M., Skinner, C. S., Rimer, B. K., \& Siegler, I. C. (2001). Depressive symptoms, menopausal status, and climacteric symptoms in women at midlife. Psychosomatic Medicine, 63, 603-608.

Bromberger, J. T., Meyer P. M., Howard M., Kravitz, D. O., Sommer, B., Cordal, A., Powell, L., Ganz, P. A., \& Sutton-Tyrrel, K. (2001). Psychological distress and natural menopause: A multiethnic community study. Research, 91 (9), $1435-1442$.

Bush, C. M., \& Zonderman, A. B. (1994). Menopausal transition and psychological distress in a nationally representative sample: Is menopause associated with psychological stress? Journal of Ageing \& Health, 6 (2), 209-229.

Cain, V. S., Johannes, C. B., Avis, N. E., Mohr, B., Skurnick, J., \& Ory, M. (2003). Sexual functioning and practices in a multi-ethnic study of midlife women: Baseline results from SWAN. The Journal of Sex Research, 40 (3), 266-276.

Deutsch, H. (1967). La psychologie des femmes: Étude psychanalytique. Paris: Presses Universitaires de France.

First, M. B., Spitzer, R. L., Gibbon, M., \& Williams, J. B. W. (1997). User's guide for the structured clinical interview for DSM-IV axis I disorders - Clinician version (SCID-CV). Washington, DC: American Psychiatric Press. 
Freud, S. (1978). Types of onset on neurosis. The standard edition of the complete psychological works of Sygmund Freud, Vol. XII (pp. 231-238). London: The Hogarth Press and the Institute of Psycho-Analysis (Publicado originalmente em 1912).

Gannon, L., \& Ekstrom, B. (1993). Attitudes towards menopause. The influence of sociocultural paradigms. Psychology of Women Quaterly, 17, 275-288.

Gonyea, J. G. (1998). Midlife and menopause: Uncharted territories for baby boomer women. Generations, 22 (1), 87-90.

Greendale, G. A., Lee, N. P., \& Arriola, E. R. (1999). The menopause. The Lancet, 353 (13), 571-580.

Hunter, M. S. (1990). Psychological and somatic experience of menopause: a prospective study. Psychossomatic Medicine, 52 (3), 357-367.

Hunter, M., \& O'Dea, I. (2001). Cognitive appraisal of the menopause: The menopause representations questionnaire (RRQ). Psychology, Health \& Medicine, $6(1), 65-76$.

Huffman, S. B., \& Myers, J. E. (1999). Counseling women in midlife: An integrative approach to menopause. Journal of Counselling \& Development, 77, 258$-266$.

Hulka, B. S., \& Meirik, O. (1996). Research on the menopause. Maturitas, 23, 109-112 .

Idrissi el Ganouni, M. C. L. C. (2002). Sexualité feminine à la ménopause. Tese de doutoramento não publicada. Paris: Université Paris XIII Villetaneuse.

Im, E., \& Meleis, A. I. (2000). Meanings of menopause to Korean immigrant women. Western Journal of Nursing Research, 22 (1), 84-102.

Kittel, L. A., Mansfield, P. K., \& Voda, A. M. (1998). Keeping up appearances: The basic social process of the menopausal transition. Qualitative Health Research, 8 (5), 618-633.

Kurpius, S. E., Nicpon, M. F., \& Maresh, S. E. (2001). Mood, marriage, and menopause. Journal of Counseling Psychology, 48 (1), 77-84.

Laznik, M. C. (2003). O complexo de Jocasta. A feminilidade e a sexualidade sob o prisma da menopausa. Rio de Janeiro: Companhia de Freud.

Lee, K. H. (1997). Korean urban women's experience of menopause: New life. Health Care for Women International, 18 (2), 19-29.

Lockely, M. (1991). Contested meanings of the menopause. Lancet, 337 (8752), 1270-1273.

Matthews, K. A., Wing, R. R., Kuller, L. H., Meilahn, E. N., Kelsey, S. F., Costello, E. J., \& Caggiula, A. W. (1990). Influences of natural menopause on psychological characteristics and symptoms of middle-aged healthy women. Journal of Consulting and Clinical Psychology, 58 (3), 345-351.

McGrath, E., Keita, G. P., Strickland, B. R., \& Russo, N. F. (1995). Women and depression. Risk factors and treatment issues. Final Report of the American Psychological Association's National Task Force on Women and Depression. Washington, DC: American Psychological Association.

McKinlay, S. M. (1996). The normal menopause transition: An overview. Maturitas, 23, 137-145. 
McKinlay, J. B., McKinlay, S. M., \& Brambilla, D. J. (1987). Health status and utilization behavior associated with menopause. American Journal of Epidemiology, 125, 110-121.

Mendell, D. (1988). A study of menopausal women in analytical treatment. In J. Offerman-Zuckerberg (Ed.), Critical passages in the life of a woman. A psychodynamic perspective. (pp. 185-197). Nova Iorque: Plenum Publishing Corporation.

Mingo, C., Herman, C. J., \& Jasperse, M. (2000). Women's stories: Ethnic variations in women's attitudes and experiences of menopause, hysteroctomy, and hormone replacement therapy. Journal of Women's Health \& Gender-Base Medicine, 9 (2), 27-38.

Northrup, C. (2000). Corpo de mulher, sabedoria de mulher. Lisboa: Sinais de Fogo.

Polit, D. F. \& LaRocco, S. A. (1980). Social and psychological correlates of menopausal symptoms. Psychosomatic Medicine, 42 (3), 335-345.

Rostosky, S. S., \& Travis, C. B. (1996). Menopause research and the dominance of the biomedical model 1984-1994. Psychology of Women Quaterly, 20, 285-312 .

Sampselle, C. M., Harris, V., Harlow, S. D., \& Sowers, M. F. (2002). Midlife development and menopause in African American and Caucasian women. Health Care for Women International, 23, 351-363.

Sheehy, G. (1994). The silent passage. Menopause. London: Harper Collins.

Stephens, C. (2001). Women's experience at the time of menopause: Accounting for biological, cultural and psychological embodiment. Journal of Health Psychology, $6(6), 651-663$. 